\title{
Integrability in Higher Dimensions: A model with exact hopfion solutions.
}

\author{
O. Babelon* \\ Laboratoire de Physique Theorique et Hautes Energies \\ Universités Paris VI-Paris VII \\ Boîte 126, Tour 16, $1^{\mathrm{er}}$ étage \\ 4 Place Jussieu, F-75252, Paris CEDEX 05, France \\ E-mail babelon@lpthe.jussieu.fri
}

\section{L.A. Ferreira}

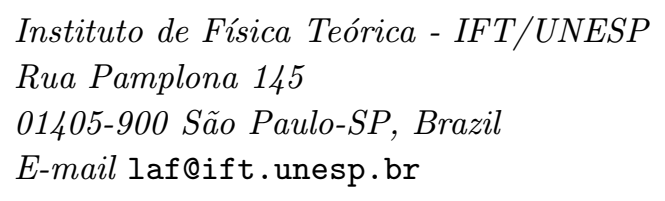

ABstract: We review concepts of integrability in higher dimensions and apply them to construct Lorentz invariant field theories with an infinite number of local conserved currents.

\section{Non Abelian Stokes theorem.}

One of the basic ingredients of two-dimensional integrable models is the so-called LaxZakharov-Shabat zero curvature condition

$$
\partial_{\mu} A_{\nu}-\partial_{\nu} A_{\mu}+\left[A_{\mu}, A_{\nu}\right]=0 \quad \mu, \nu=0,1
$$

In $1+1$ dimensions the relation $\left(\begin{array}{l}1 \\ 1\end{array}-1,1\right)$ is a conservation law, since it is a sufficient condition for the path ordered integral

$$
W(\Gamma)=P_{\Gamma} \exp \int_{\Gamma} A_{\mu} d x^{\mu}
$$

to be independent of the path $\Gamma$, as long as the end points are kept fixed. Consequently, by choosing appropriate boundary conditions at space infinity (or taking space to be a circle and so space-time a cylinder) one gets that the quantities

$$
Q_{N} \equiv \operatorname{Tr}\left(P \exp \int_{\text {space }} A_{x} d x\right)^{N}
$$

${ }^{*}$ Speaker. 
are conserved in time. These are the conservation laws responsible for the integrability of a two dimensional theory possessing the zero curvature representation (i1.

In $2+1$ dimension, it is natural to replace line integrals by surface integrals:

$$
V(\Sigma)=P_{\Sigma} \exp \int_{\Sigma} B_{\mu \nu} d x^{\mu} \wedge d x^{\nu}
$$

We face the problem of defining $P_{\Sigma}$. The answer given in [i] $\left[\begin{array}{l}1 \\ ]\end{array}\right]$ is as follows: scan $\Sigma$ with a family of loops $\gamma(\tau)$ with base point $x_{0}$, each point of $\Sigma$ belonging to exactly one loop $\gamma$. Hence each point $x$ of $\Sigma$ can be localised by choosing the curve $\gamma$ and a point with coordinate $\sigma$ on it. Introduce a gauge potential $A$, and define

$$
\mathcal{A}=\int_{0}^{2 \pi} d \sigma B_{\mu \nu}^{W}(\sigma) \frac{d x^{\mu}}{d \sigma} \delta x^{\nu}(\sigma), \quad B_{\mu \nu}^{W}(\sigma)=W^{-1}(\sigma) B_{\mu \nu}(\sigma) W(\sigma)
$$

where $W(\sigma)$ is defined as in eq.(1 1.2 .2$)$ using $A$ and the path $\gamma$, joining the point $x$ to the base point $x_{0}$. Then we solve the differential equation

$$
\frac{d V(\tau)}{d \tau}=V(\tau) \mathcal{A}\left(\frac{d x^{\nu}}{d \tau}\right), \quad V(0)=1
$$

and define

$$
\left.V(\Sigma) \equiv V(\tau)\right|_{\tau=1}
$$

The quantity $\mathcal{A}$ can be viewed as a connection on loop space. Its curvature is:

$$
\mathcal{C}=\delta \mathcal{A}+\mathcal{A} \wedge \mathcal{A}
$$

The flux of $B$ will be surface independent if $\mathcal{C}$ vanishes. Therefore, imposing apropriated boundary conditions, one can construct conserved quantities when $\mathcal{C}$ vanishes in a similar way as in (1) (1) for the $1+1$ case. See [i] for details on the construction of the conserved charges.

For infinitesimal loops around $x_{0}$, vanishing of $\mathcal{C}$ reduces to the local conditions $\left[\begin{array}{l}1 \\ 1\end{array}\right.$, ind

$$
\omega=0, \quad[\nu, B]=0
$$

where

$$
\omega=d B+A \wedge B, \quad \nu=d A+A \wedge A-B
$$

Note that we have the Bianchi type identities:

$$
d \nu+A \wedge \nu=-[\omega, \cdot], \quad d \omega+A \wedge \omega=[\nu, B]
$$

The two form $\nu$ is called the fake curvature. In the setting we consider below, the local equations (1) are sufficient to ensure the vanishing of $\mathcal{C}$, even for big loops. 


\section{Integrable models and submodels.}

To get independence upon the way $\Sigma$ is scanned by the loops $\gamma$, we choose the potential $A$ to be a pure gauge:

$$
F_{\mu \nu}=0 \Longrightarrow \nu=-B
$$

To ensure $\mathcal{C}=0$ we furthermore require

$$
\begin{gathered}
\omega=d B+A \wedge B=0 \\
B \text { in an abelian ideal of } \mathcal{G} \Longrightarrow[\nu, B]=0
\end{gathered}
$$

Introducing the dual form of $B$

$$
B_{\mu}^{*}=\epsilon_{\mu \nu \lambda} B^{\nu \lambda}
$$

the condition $(\overline{2}, \overline{1} \cdot 1)$ becomes

$$
D_{A}^{\mu} B_{\mu}^{*}=0
$$

This yields conserved currents, because setting

$$
J_{\mu}=W^{-1} B_{\mu}^{*} W
$$

eq. $(\overline{2} \cdot \overline{2} . \overline{3})$ reads

$$
\partial^{\mu} J_{\mu}=0
$$

The number of conserved currents is the dimension of the abelian ideal of $\mathcal{G}$

Since, the potential $B$ has to live on an Abelian ideal we consider a Poincaré type non-semisimple Lie algebra $\mathcal{G}=T+P$, satisfying

$$
[T, T] \subset T \quad[T, P] \subset P \quad[P, P]=0
$$

where $T$ is a Lie algebra and $P$ a representation of it. In order for the model to be integrable we need an infinite number of conserved currents of the form (2.2. representation $P$ has to be infinite dimensional. In order to get that, we shall use the Schwinger's construction. To implement these ideas, start from $\operatorname{sl}(2)$, and introduce its $\lambda$ $-\bar{\lambda}$ realization:

$$
T_{+} \equiv \lambda \frac{d}{d \bar{\lambda}}, \quad T_{-} \equiv \bar{\lambda} \frac{d}{d \lambda}, \quad T_{3} \equiv \frac{1}{2}\left(\lambda \frac{d}{d \lambda}-\bar{\lambda} \frac{d}{d \bar{\lambda}}\right)
$$

One can check that these operators satisfy the $s l(2)$ algebra, namely

$$
\left[T_{3}, T_{ \pm}\right]= \pm T_{ \pm} ; \quad\left[T_{+}, T_{-}\right]=2 T_{3}
$$

The states of the representations corresponding to such realization are functions of $\lambda$ and $\bar{\lambda}$. The action of the operators are given by

$$
\begin{aligned}
T_{3} \lambda^{p} \bar{\lambda}^{q} & =\frac{p-q}{2} \lambda^{p} \bar{\lambda}^{q} \\
T_{+} \lambda^{p} \bar{\lambda}^{q} & =q \lambda^{p+1} \bar{\lambda}^{q-1} \\
T_{-} \lambda^{p} \bar{\lambda}^{q} & =p \lambda^{p-1} \bar{\lambda}^{q+1}
\end{aligned}
$$


Notice from (2.6. $)$ that the action of $T_{3}, T_{ \pm}$leaves the sum of the powers of $\lambda$ and $\bar{\lambda}$ invariant. Therefore, one can construct irreducible representations by considering the states

$$
|(p, q), m\rangle \equiv \lambda^{p+m} \bar{\lambda}^{q-m}
$$

with $m \in \mathbb{Z}$ and $(p, q)$ being any pair of numbers (real or even complex). Then

$$
\begin{aligned}
& T_{3}|(p, q), m\rangle=\left(\frac{p-q}{2}+m\right)|(p, q), m\rangle \\
& T_{+}|(p, q), m\rangle=(q-m)|(p, q), m+1\rangle \\
& T_{-}|(p, q), m\rangle=(p+m)|(p, q), m-1\rangle
\end{aligned}
$$

On the subspace with fixed $(p+q)$, the Casimir operator acts as:

$$
\left(T_{3}^{2}+\frac{1}{2}\left(T_{+} T_{-}+T_{-} T_{+}\right)\right)|(p, q), m\rangle=s(s+1)|(p, q), m\rangle, \quad s=\frac{1}{2}(p+q)
$$

The parameter $s$ is the spin of the representation. Take $\mathcal{G}$ be the semi-direct product of $T=\operatorname{sl}(2)$ and the abelian algebra $P=\operatorname{Func}(\lambda, \bar{\lambda})$.

$$
A \in \operatorname{sl}(2), \text { and } B \in \operatorname{Func}(\lambda, \bar{\lambda}) \simeq\left\{\lambda^{p} \bar{\lambda}^{q}\right\}
$$

We consider models with one complex scalar field $u$. Introduce the group element

$$
W=\frac{1}{\sqrt{1+|u|^{2}}}\left(\begin{array}{cc}
1 & i u \\
i u^{*} & 1
\end{array}\right)=e^{i u T_{+}} e^{\varphi T_{3}} e^{i u^{*} T_{-}}
$$

with $\varphi \equiv \log \left(1+u u^{*}\right)$. One can check that

$$
W^{-1} f(\lambda, \bar{\lambda}) W=f\left(\frac{\lambda-i u^{*} \bar{\lambda}}{\sqrt{1+u u^{*}}}, \frac{\bar{\lambda}-i u \lambda}{\sqrt{1+u u^{*}}}\right)
$$

Define the flat potential $A_{\mu}$ by

$$
A_{\mu}=-\partial_{\mu} W W^{-1}
$$

and the field $B_{\mu}^{(s) *}=\epsilon_{\mu \nu \lambda} B^{\nu \lambda}$

$$
\begin{aligned}
A_{\mu} & \equiv \frac{1}{1+|u|^{2}}\left(-i \partial_{\mu} u \lambda \frac{d}{d \bar{\lambda}}-i \partial_{\mu} u^{*} \bar{\lambda} \frac{d}{d \lambda}+\left(u \partial_{\mu} u^{*}-u^{*} \partial_{\mu} u\right) \frac{1}{2}\left(\lambda \frac{d}{d \lambda}-\bar{\lambda} \frac{d}{d \bar{\lambda}}\right)\right) \\
B_{\mu}^{*(s)} & \equiv \frac{1}{1+|u|^{2}}\left(\mathcal{K}_{\mu} \lambda^{s+1} \bar{\lambda}^{s-1}-\mathcal{K}_{\mu}^{*} \lambda^{s-1} \bar{\lambda}^{s+1}\right)
\end{aligned}
$$

where $\mathcal{K}_{\mu}$ is a functional of $u, u^{*}$ and their derivatives. Notice that we have chosen $B_{\mu}^{(s)}$ to live in a representation where $p=q=s$. Performing the calculation one gets

$$
\begin{aligned}
D^{\mu} B_{\mu}^{*(s)} & =\partial^{\mu} B_{\mu}^{*(s)}+\left[A^{\mu}, B_{\mu}^{(s)}\right] \\
& =\frac{1}{\left(1+|u|^{2}\right)^{2}}\left(\left(-i(s-1) \partial^{\mu} u \mathcal{K}_{\mu}\right) \lambda^{s+2} \bar{\lambda}^{s-2}\right. \\
& +\left(i(s-1) \partial^{\mu} u^{*} \mathcal{K}_{\mu}^{*}\right) \lambda^{s-2} \bar{\lambda}^{s+2} \\
& +\left(-i(s+1)\left(\partial^{\mu} u^{*} \mathcal{K}_{\mu}-\partial^{\mu} u \mathcal{K}_{\mu}^{*}\right)\right) \lambda^{s} \bar{\lambda}^{s} \\
& +\left(\left(\left(1+|u|^{2}\right) \partial^{\mu} \mathcal{K}_{\mu}-2 u^{*} \partial^{\mu} u \mathcal{K}_{\mu}\right)\right) \lambda^{s+1} \bar{\lambda}^{s-1} \\
& \left.-\left(\left(\left(1+|u|^{2}\right) \partial^{\mu} \mathcal{K}_{\mu}^{*}-2 u \partial^{\mu} u^{*} \mathcal{K}_{\mu}^{*}\right)\right) \lambda^{s-1} \bar{\lambda}^{s+1}\right)
\end{aligned}
$$


Therefore, the zero curvature implies the following: For $s \neq 1,-1$ we get the equations of motion

$$
\begin{aligned}
\partial^{\mu} \mathcal{K}_{\mu} & =0 \\
\partial^{\mu} u \mathcal{K}_{\mu} & =0 \\
\partial^{\mu} u^{*} \mathcal{K}_{\mu}-\partial^{\mu} u \mathcal{K}_{\mu}^{*} & =0
\end{aligned}
$$

These conditions ensure that

$$
D^{\mu} B_{\mu}^{*(s)}=0 \quad \forall s
$$

Hence, such a model has an infinite number of conserved currents.

Let $K_{\mu}$ be the tensor

$$
K_{\mu}=\left(\partial^{\nu} u^{*} \partial_{\nu} u\right) \partial_{\mu} u-\left(\partial^{\nu} u \partial_{\nu} u\right) \partial_{\mu} u^{*}
$$

Then, for any real function $\mathcal{F}\left(u, u^{*}, \partial u, \partial u^{*}\right)$

$$
\mathcal{K}_{\mu}=\mathcal{F} K_{\mu}
$$

satisfies identically the conditions eq. $(2 \cdot 13,2,14)$. So the model reduces to a single equation eq. (2.12) which reads:

$$
\partial^{\mu}\left(\mathcal{F} K_{\mu}\right)=0
$$

Remark 1. Setting $\mathcal{K}_{\mu}=\partial_{\mu} u$, we get the equations

$$
\partial^{2} u=0 \quad(\partial u)^{2}=0
$$

which define a submodel of the $C P^{1}$ model:

$$
\left(1+|u|^{2}\right) \partial^{2} u=2 u^{*}(\partial u)^{2}
$$

This submodel has an infinite number of conserved currents.

Remark 2. Similarly, setting $\mathcal{K}_{\mu}=L_{\mu}$ with

$$
L_{\mu}=m^{2} \partial_{\mu} u-\frac{4}{e^{2}} \frac{K_{\mu}}{\left(1+|u|^{2}\right)^{2}}
$$

where $K_{\mu}$ is as above, we get

$$
\partial^{\mu}\left(\Delta \partial_{\mu} u\right)=0 \quad \partial_{\mu} u \partial^{\mu} u=0
$$

where

$$
\Delta=m^{2}-\frac{4}{e^{2}} \frac{\partial_{\nu} u \partial^{\nu} u^{*}}{\left(1+|u|^{2}\right)^{2}}
$$

which is a submodel of the Skyrme-Faddeev model:

$$
\left(1+|u|^{2}\right) \partial^{\mu} L_{\mu}-2 u^{*} \partial^{\mu} u L_{\mu}=0
$$

In this note we restrict ourselves to models and will not consider submodels. 


\section{Lagrangian}

The question then arises to know what are the models eq. (2.15) which can be derived from an action principle

$$
S=\int d^{4} x \mathcal{L}
$$

The Lagrangian is local, Lorentz invariant and depends only on first order derivatives. Hence it is of the form $\mathcal{L}=\mathcal{L}\left(V, V^{*}, W, u, u^{*}\right)$ where $V=\partial^{\mu} u \partial_{\mu} u, V^{*}=\partial^{\mu} u^{*} \partial_{\mu} u^{*}$, $W=\partial^{\mu} u \partial_{\mu} u^{*}$. So, we want

$$
\partial^{\mu}\left(-2 \frac{\partial \mathcal{L}}{\partial V} \partial_{\mu} u-\frac{\partial \mathcal{L}}{\partial W} \partial_{\mu} u^{*}\right)+\frac{\partial \mathcal{L}}{\partial u}=\Lambda \partial^{\mu}\left(\mathcal{F} K_{\mu}\right)+\Phi \partial^{\mu}\left(\mathcal{F} K_{\mu}^{*}\right)
$$

The functions $\mathcal{L}$ and $\mathcal{F}$ should be real. The solution is that

$$
\mathcal{L}=\mathcal{L}\left(\frac{h^{2}}{2 f^{2}}\right), \quad h^{2}=h_{\mu \nu} h^{\mu \nu}=2\left(V V^{*}-W^{2}\right)
$$

where

$$
h_{\mu \nu}=\partial_{\mu} u \partial_{\nu} u^{*}-\partial_{\nu} u \partial_{\mu} u^{*}
$$

and $f$ is a function of $u$ and $u^{*}$ only $f=f\left(u, u^{*}\right)$. The equations of motion are

$$
\partial^{\mu}\left(\mathcal{F} K_{\mu}\right)=0
$$

where

$$
\mathcal{F}=\frac{1}{f} \mathcal{L}^{\prime}, \quad K_{\mu}=h_{\mu \nu} \partial^{\nu} u
$$

The particularly nice models are obtained by choosing

$$
\mathcal{L}=\left(\frac{h^{2}}{2 f^{2}}\right)^{3 / 4}
$$

This has the advantage of circumventing Derrick's theorem, so that stable static solutions may exist. The equations of motion read

$$
\mathcal{E} \equiv\left(h_{\mu \nu} \partial^{\nu} u \partial^{\mu} h^{2}-4 h^{2} \partial^{\mu} h_{\mu \nu} \partial^{\nu} u\right)-\left(h^{2}\right)^{2} \partial_{u^{*}} \log f=0
$$

In the case where the target space is the sphere $S^{2}$ we have $f=\left(1+u u^{*}\right)^{2}$ where $u$ and $u^{*}$ are the stereographic projection coordinates on $S^{2}$ :

$$
\vec{n}=\frac{1}{1+u u^{*}}\left(u+u^{*},-i\left(u-u^{*}\right),-1+u u^{*}\right), \quad \vec{n}^{2}=1, \quad u=\frac{n_{x}+i n_{y}}{1-n_{z}}
$$

For finite energy static solutions, $\vec{n}$ tends to a constant at infinity. Such solutions can be viewed as maps from $S^{3} \rightarrow S^{2}$, hence are classified by the Hopf invariant. So, in this case we can have solutions with non trivial topological charges. 


\section{Conserved currents}

These models have an infinite number of conserved currents. For any $s$, the current

$$
J_{\mu}^{(s)}=W^{-1} B_{\mu}^{(s)} W
$$

is conserved. We see from eq.(2).10

$$
B_{\mu}^{(s)}=(\lambda \bar{\lambda})^{(s+1)} B_{\mu}^{(-1)}
$$

If we consider a general $B_{\mu}=\sum_{s} b_{s} B_{\mu}^{(s)}$, we have $B_{\mu}=b(\lambda \bar{\lambda}) B_{\mu}^{(-1)}$, where $b(z)=\sum_{s} b_{s} z^{s+1}$ is essentially an arbitrary function. At the level of currents, this means

$$
J_{\mu}=b\left(\frac{\left(\lambda-i u^{*} \bar{\lambda}\right)(\bar{\lambda}-i u \lambda)}{1+u u^{*}}\right) J_{\mu}^{(-1)}
$$

We can write this in the form:

$$
J_{\mu}=\mathcal{K}_{\mu} \frac{\delta G}{\delta u}-\mathcal{K}_{\mu}^{*} \frac{\delta G}{\delta u^{*}}
$$

where

$$
G=i \int^{v\left(u, u^{*}\right)} \frac{d z}{z^{2}} b(z), \quad v\left(u, u^{*}\right)=\frac{\left(\lambda-i u^{*} \bar{\lambda}\right)(\bar{\lambda}-i u \lambda)}{1+u u^{*}}
$$

If we now introduce the conjugate momentum of the field $u$,

$$
\pi=\frac{\partial \mathcal{L}}{\partial \dot{u}}=\frac{1}{f} \mathcal{K}_{0}^{*}
$$

the conserved charges become

$$
Q_{G}=i \int d^{3} x f\left(\pi^{*} \frac{\delta G}{\delta u}-\pi \frac{\delta G}{\delta u^{*}}\right)
$$

If we impose the Poisson bracket

$$
\{\pi(x), u(y)\}=\delta(x-y)
$$

we immediately get

$$
\left\{Q_{G}, u\right\}=-i f \partial_{u^{*}} G
$$

The meaning of the conserved currents is now clear: $Q_{G}$ generates area preserving diffeomorphisms. Indeed the Lagrangian reads

$$
\mathcal{L}=\mathcal{L}\left(A_{\mu \nu} A^{\mu \nu}\right)
$$

where

$$
A=\frac{1}{f} h_{\mu \nu} d x^{\mu} \wedge d x^{\nu}=\frac{1}{f} d u \wedge d u^{*}
$$

The form $A$ is the pullback of an area form on target space. The action is invariant under area preserving diffeomorphisms. The conserved currents are the associated Noether currents $[\overline{3} \overline{3}]$. 


\section{Conformal symmetries}

The special case of the models defined in eq.(3.1) brings up the possibility of extra symmetries related to scale transformations. We apply S. Lie theory. To say that $x \rightarrow x+\xi$ is a symmetry of a differential equation $\mathcal{E}\left(x, u(x), u^{\prime}(x)\right)=0$ means that if $u(x)$ is a solution so is $u(x-\xi)$, i.e.,

$$
\begin{array}{ll} 
& \mathcal{E}\left(x, u(x-\xi), \frac{d}{d x} u(x-\xi)\right)=0 \\
\text { or } \quad & \mathcal{E}\left(x, u(x-\xi), u^{\prime}(x-\xi)-\xi^{\prime} u^{\prime}(x-\xi)\right)=0 \\
\text { or } \quad & \mathcal{E}\left(x+\xi, u(x), u^{\prime}(x)-\xi^{\prime} u^{\prime}(x)\right)=0 \\
\text { or } \quad & \left(\xi \partial_{x}-\xi^{\prime} u^{\prime} \partial_{u^{\prime}}\right) \mathcal{E}\left(x, u, u^{\prime}\right)=0
\end{array}
$$

More generally, if the vector field of the symmetry reads

$$
V=\sum_{\mu} \xi^{\mu} \partial_{\mu}
$$

the first prolongations are

$$
\begin{gathered}
\delta u=0 \\
\delta \partial_{\mu} u=-\partial_{\mu} \xi^{\nu} \partial_{\nu} u \\
\delta \partial_{\mu} \partial_{\nu} u=-\left(\partial_{\mu} \partial_{\nu} \xi^{\rho}\right) \partial_{\rho} u-\partial_{\mu} \xi^{\rho} \partial_{\rho} \partial_{\nu} u-\partial_{\nu} \xi^{\rho} \partial_{\rho} \partial_{\mu} u
\end{gathered}
$$

It follows that

$$
\delta h^{2}=-2\left(\partial^{\mu} \xi^{\rho}+\partial^{\rho} \xi^{\mu}\right) h_{\rho \nu} h_{\mu}{ }^{\nu}
$$

To have any chance to get an invariance of the equations of motion, we require:

$$
\partial^{\mu} \xi^{\nu}+\partial^{\nu} \xi^{\mu}=2 D \eta^{\mu \nu}
$$

where $D$ is the common value of $\partial_{\mu} \xi^{\mu}$ (no summation). Then, we have

$$
\delta h^{2}=-4 D h^{2}
$$

So that

$$
\delta \mathcal{E}=-8 D \mathcal{E}-4(d-3) \partial^{\mu} D h_{\mu \nu} \partial^{\nu} u h^{2}
$$

where $\mathcal{E}=0$ is the equation of motion eq. (3.2.2). Hence

- If $d=3$, i.e. for the static theory, we have a symmetry of the equations of motion for all $D$. The structure of eqs. $\left(\left[\begin{array}{l}0 \\ {[}\end{array} .11\right)\right.$ is such however that $D$ has to be a linear function of $x^{\mu}$. We get translations, rotations, dilatation and a special set of vectors $V^{(i)}$ with components

$$
V^{(i) j}=x^{i} x^{j}-\frac{1}{2} \eta^{i j} x^{2}
$$

This is the full conformal group in 3 dimensions.

- If $d=4$, we have a symmetry only if $\partial^{\mu} D=0$. The above special transformations are excluded and we are left with the Poincaré group plus the dilatation. 


\subsection{Target space symmetries}

This time, the symmetry we start with is of the form

$$
V=\Phi\left(u, u^{*}\right) \partial_{u}+\Phi^{*}\left(u, u^{*}\right) \partial_{u^{*}}
$$

Then

$$
\delta h_{\mu \nu}=\left(\partial_{u} \Phi+\partial_{u^{*}} \Phi^{*}\right) h_{\mu \nu} \Longrightarrow \delta h^{2}=2\left(\partial_{u} \Phi+\partial_{u^{*}} \Phi^{*}\right) h^{2}
$$

and we find easily $[\overline{5}]$

$$
\delta \mathcal{E}=\left(4 \partial_{u} \Phi+3 \partial_{u^{*}} \Phi^{*}\right) \mathcal{E}-\partial_{u^{*}} \Phi \mathcal{E}^{*}+Q\left(h^{2}\right)^{2}
$$

where

$$
\begin{aligned}
Q= & \partial_{u^{*}}\left(\partial_{u} \Phi+\partial_{u^{*}} \Phi^{*}\right)-\partial_{u} \log f \partial_{u^{*}} \Phi-\partial_{u^{*}} \log f \partial_{u^{*}} \Phi^{*} \\
& -\partial_{u} \partial_{u^{*}} \log f \Phi-\partial_{u^{*}} \partial_{u^{*}} \log f \Phi^{*}
\end{aligned}
$$

So, we have a symmetry only if $Q=0$. Setting

$$
\Phi=f \widetilde{\Phi}
$$

this condition becomes

$$
\partial_{u^{*}}\left(f\left[\partial_{u} \widetilde{\Phi}+\partial_{u^{*}} \widetilde{\Phi}^{*}\right]\right)=0
$$

Integrating once, we get (choosing the integration constant equal to 2)

$$
\partial_{u} \widetilde{\Phi}+\partial_{u^{*}} \widetilde{\Phi}^{*}=\frac{2}{f}
$$

Setting, for real $G$ and $F$ :

$$
\widetilde{\Phi}=\partial_{u^{*}}(G+i F)
$$

the equation becomes

$$
\partial_{u} \partial_{u^{*}} G=\frac{1}{f}
$$

The function $F$ has disappeared. It corresponds to area preserving diffeomorphisms. The function $G$ generates a new symmetry, not preserving the area. For the sphere, we have $f=\left(1+u u^{*}\right)^{2}$ and we find

$$
G=\log \left(1+u u^{*}\right), \quad \Longrightarrow \Phi=\left(1+u u^{*}\right) u
$$

\section{Toroidal solutions}

Let us consider the static case $d=3$. There is a nice description of conformal symmetries of Euclidean space. Just consider points of Euclidean space as spheres of radius zero! Equation of spheres is of the form

$$
\alpha \vec{x}^{2}-2 \vec{\beta} \cdot \vec{x}+\gamma=0
$$


or

$$
\left(\vec{x}-\frac{\vec{\beta}}{2 \alpha}\right)^{2}=\frac{\vec{\beta}^{2}-\alpha \gamma}{\alpha^{2}}
$$

So Euclidean space is described by

$$
\vec{\beta}^{2}-\alpha \gamma=0, \quad \vec{x}=\frac{\vec{\beta}}{2 \alpha}
$$

Any linear transformation which preserves the first condition, acts on $\vec{x}$ through the second equation. These are the conformal transformations.

Write the quadrics as

$$
\beta_{1}^{2}+\beta_{2}^{2}+\beta_{3}^{2}+\left(\frac{a \alpha-a^{-1} \gamma}{2}\right)^{2}-\left(\frac{a \alpha+a^{-1} \gamma}{2}\right)^{2}
$$

where $a$ is an arbitrary scale. From this, it is apparent that the conformal group is $O(4,1)$. It is also very obvious that there is at most only 2 commuting, compact rotations. One can be chosen as the rotation $\left(\beta_{1}, \beta_{2}\right)$, the other one as the rotation $\left(\beta_{3}, \frac{a \alpha-a^{-1} \gamma}{2}\right)$.

Transported to $\vec{x}$, the vector fields corresponding to the two rotations are

$$
\begin{aligned}
& \partial_{\theta}=x \partial_{y}-y \partial_{x} \\
& \partial_{\xi}=\frac{z x}{a} \partial_{x}+\frac{z y}{a} \partial_{y}+\frac{1}{2 a}\left(z^{2}-x^{2}-y^{2}+a^{2}\right) \partial_{z}
\end{aligned}
$$

Ansatz for solutions of the equations of motion are constructed by imposing invariance under the two commuting vector fields

$$
\begin{aligned}
{\left[\partial_{\theta}-i n\left(u \partial_{u}-u^{*} \partial_{u^{*}}\right)\right] u } & =0 \\
{\left[\partial_{\xi}-i m\left(u \partial_{u}-u^{*} \partial_{u^{*}}\right)\right] u } & =0
\end{aligned}
$$

then we have [2]

$$
u=f(\zeta) e^{i n \theta+i m \xi}
$$

where $\zeta$ is such that $\partial_{\theta} \zeta=\partial_{\xi} \zeta=0$. This invariant can be chosen as

$$
\zeta=\frac{a^{2}\left(x^{2}+y^{2}\right)}{\left(x^{2}+y^{2}+z^{2}+a^{2}\right)^{2}}
$$

Comparing with toroidal coordinates,

$$
\begin{aligned}
& x=a q^{-1} \sinh \eta \cos \theta \\
& y=a q^{-1} \sinh \eta \sin \theta \\
& z=a q^{-1} \sin \xi \\
& q=\cosh \eta-\cos \xi
\end{aligned}
$$

we find

$$
\zeta=\frac{1}{4} \tanh ^{2} \eta
$$


The two cyclic coordinates $\theta, \xi$, will disappear from the equations of motion. So we know

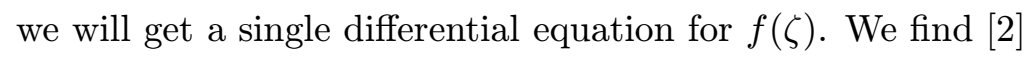

$$
f^{2}=\frac{\cosh \eta-\sqrt{n^{2} / m^{2}+\sinh ^{2} \eta}}{\sqrt{1+m^{2} / n^{2} \sinh ^{2} \eta}-\cosh \eta}
$$

The Hopf charge and the energy are

$$
Q_{H}=-n m, \quad E=(2 \pi)^{2} \sqrt{|n||m|(|n|+|m|)}
$$

\section{Flux calculation}

Under the extra target space symmetry eq. $\left(\left[\begin{array}{l}5 \\ 5\end{array} .2\right)\right.$, we have

$$
\delta \mathcal{L}=3 \mathcal{L}
$$

When $d=4$, we can compensate this variation by a dilatation to get a symmetry of the action.

$$
\delta u=\epsilon\left(1+u u^{*}\right) u, \quad \delta x^{\mu}=-3 \epsilon x^{\mu}
$$

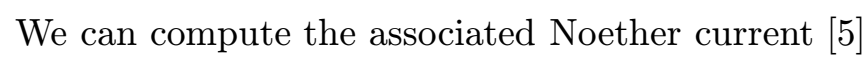

$$
J^{\mu}=-2 x^{\mu} \mathcal{L}+\left(\frac{h^{2}}{2 f^{2}}\right)^{-1 / 4} \frac{1}{f^{2}}\left(h^{\mu \nu}\left(\Phi \partial_{\nu} u^{*}-\Phi^{*} \partial_{\nu} u\right)-3 x^{\rho} h_{\rho \nu} h^{\nu \mu}\right)
$$

This current is conserved. Then

$$
\frac{d Q}{d t}=\int d^{3} x \partial_{0} J^{0}=-\int d^{3} x \partial_{i} J^{i}=-\int d \Sigma^{i} J_{i}
$$

The surface term does not vanish in general, and so such the charge is not conserved. For instance, for static configurations we have that

$$
\frac{d Q}{d t}=-\int d^{3} x \mathcal{L}=E \equiv \text { static energy }
$$

Hence the energy of the toroidal solutions eq.( $\left(\overline{6}_{1} \cdot \overline{1}_{j}\right)$ can be written as a flux of a current concentrated along a small tube around the $z$-axis.

\section{Conclusion.}

The ideas of [i] to extend in higher dimensions some notions of integrability, do provide us with models with an infinite number of conserved currents. It turned out that these currents, in the case of the models discussed here, are associated to an invariance of the Lagrangian under area preserving diffeomorphisms. It is intriguing that they do not play an important role in the construction of the static solutions of the equations of motion. That role is played instead by the conformal symmetry of the static equations of motion, which leads to the construction of the relevant Ansatz. It remains to see the role of the conserved currents in the scatterring processes of these solutions. 


\section{References}

[1] O. Alvarez, L.A. Ferreira, J. Sanchez Guillen, A new approach to integrable theories in any dimension. ; Nucl.Phys. B529 (1998) 689-736, hep-th/9710147.

[2] H. Aratyn, L.A. Ferreira, A.H. Zimerman Exact static soliton solutions of $3+1$ dimensional integrable theory with nonzero Hopf numbers.; Phys.Rev.Lett. 83 (1999) 1723-1726, hep-th/9905079.

[3] L.A. Ferreira, A.V. Razumov, Hopf solitons and area preserving diffeomorphisms of the sphere.; Lett.Math.Phys. 55 (2001) 143-148, hep-th/0012176

[4] L. Breen and W. Messing, Differential Geometry of Gerbes, math.AG/0106083 R. Attal, Combinatorics of Non-Abelian Gerbes with Connection and Curvature, math-ph/0203056

J. C. Baez, Higher Yang-Mills Theory, hep-th/0206130.

[5] O. Babelon, L. Ferreira Integrability and Conformal Symmetry in Higher Dimensions: a Model with Exact Hopfion Solutions. hep-th/0210154 